\title{
MAPPINGS ON MATRICES: INVARIANCE OF FUNCTIONAL VALUES OF MATRIX PRODUCTS
}

\author{
JOR-TING CHAN ${ }^{\star}$, CHI-KWONG LI and NUNG-SING SZE
}

(Received 8 December 2004; revised 9 May 2005)

Communicated by J. Du

\begin{abstract}
Let $\mathcal{M}_{n}$ be the algebra of all $n \times n$ matrices over a field $\mathbb{F}$, where $n \geq 2$. Let $\mathcal{S}$ be a subset of $\mathcal{M}_{n}$ containing all rank one matrices. We study mappings $\phi: \mathcal{S} \rightarrow \mathcal{M}_{n}$ such that $F(\phi(A) \phi(B))=F(A B)$ for various families of functions $F$ including all the unitary similarity invariant functions on real or complex matrices. Very often, these mappings have the form $A \mapsto \mu(A) S\left(\sigma\left(a_{i j}\right)\right) S^{-1}$ for all $A=\left(a_{i j}\right) \in \mathcal{S}$ for some invertible $S \in \mathcal{M}_{n}$, field monomorphism $\sigma$ of $\mathbb{F}$, and an $\mathbb{F}^{*}$-valued mapping $\mu$ defined on $\mathcal{S}$. For real matrices, $\sigma$ is often the identity map; for complex matrices, $\sigma$ is often the identity map or the conjugation map: $z \mapsto \bar{z}$. A key idea in our study is reducing the problem to the special case when $F: \mathcal{M}_{n} \rightarrow\{0,1\}$ is defined by $F(X)=0$, if $X=0$, and $F(X)=1$ otherwise. In such a case, one needs to characterize $\phi: \mathcal{S} \rightarrow \mathcal{M}_{n}$ such that $\phi(A) \phi(B)=0$ if and only if $A B=0$. We show that such a map has the standard form described above on rank one matrices in $\mathcal{S}$.
\end{abstract}

2000 Mathematics subject classification: primary 15A04, 15A60, $15 \mathrm{~A} 18$.

Keywords and phrases: zero product preservers, unitary similarity invariant functions.

\section{Introduction}

Let $\mathcal{M}_{n}$ be the algebra of all $n \times n$ matrices over a field $\mathbb{F}$, where $n \geq 2$. There has been considerable interest in studying preserver problems on $\mathcal{M}_{n}$, which concern the characterization of mapping $\phi: \mathcal{M}_{n} \rightarrow \mathcal{M}_{n}$ leaving invariant a set, a function, or a relation. In early studies, the mappings were often assumed to be linear; the quest to describe these mappings is collectively called linear preserver problems; see $[11,15]$.

This research was supported by the Hong Kong RCG CERG grant HKU 7007/03P.

$\mathrm{Li}$ is an honorary professor of the Heilongiiang University, and also an honorary professor of the University of Hong Kong. His research was partially supported by a USA NSF grant.

The third author was supported by a HKU studentship.

(C) 2006 Australian Mathematical Society 1446-7887/06 $\$$ A2.00+0.00 
Recently, researchers have considered additive preservers, multiplicative preservers, or preservers under other milder assumptions, see [20]. For example, given a function $F: \mathcal{M}_{n} \rightarrow \mathbb{F}, \mathcal{S} \subseteq \mathcal{M}_{n}$, and $\mathcal{T} \subseteq \mathbb{F}$, researchers characterize $\phi: \mathcal{S} \rightarrow \mathcal{M}_{n}$ such that

$$
F(\phi(A)+\mu \phi(B))=F(A+\mu B) \text { for all } A, B \in \mathcal{S}, \mu \in \mathcal{T},
$$

and particular attention is on the cases when $\mathcal{T}=\{1\}$ or $\{-1\}$; see $[2,4,5,8,16]$. Another problem is to characterize $\phi: \mathcal{S} \rightarrow \mathcal{M}_{n}$ such that

$$
F(\phi(A) \phi(B))=F(A B) \text { for all } A, B \in \mathcal{S} ;
$$

see $[6,13,14,21]$. In this paper, we consider this problem for various functions $F$ including all the unitary similarity invariant norms on real or complex matrices. Very often, these mappings have the form

$$
A \mapsto \mu(A) S\left(\sigma\left(a_{i j}\right)\right) S^{-1} \text { for all } A=\left(a_{i j}\right) \in \mathcal{S}
$$

for some invertible $S \in \mathcal{M}_{n}$, field monomorphism $\sigma$ of $\mathbb{F}$, and an $\mathbb{F}^{*}$-valued mapping $\mu$ defined on $\mathcal{S}$. For real matrices, $\sigma$ is often the identity map; for complex matrices, $\sigma$ is often the identity map or the conjugation map: $z \mapsto \bar{z}$. We do not require $A B \in \mathcal{S}$ even if $A, B \in \mathcal{S}$ in our setting. However, we do require that $\mathcal{S}$ contains all rank one idempotents or rank one matrices.

In studying preservers, one may develop special techniques to deal with a specific problem, one may develop a general technique to treat a class of problems, or one may obtain a basic preserver result so that other preserver problems can be reduced to it. For instance, many linear preserver problems can be reduced to rank one preservers or nilpotent preservers. In our study, many general problems are reduced to the special case when $F: \mathcal{M}_{n} \rightarrow\{0,1\}$ is defined by

$$
F(X)= \begin{cases}0 & \text { if } X=0 \\ 1 & \text { otherwise }\end{cases}
$$

In such a case, one needs to characterize mappings $\phi: \mathcal{S} \rightarrow \mathcal{M}_{n}$ such that

$$
\phi(A) \phi(B)=0 \quad \text { if and only if } A B=0 .
$$

We will call such mappings zero product preservers. Many researchers have study bijective zero product preservers; see Section 2 for more details. In our study, the bijectivity assumption is not required. We show that if $n \geq 3$, then for every zero product preserver $\phi$, there exist an invertible $S \in \mathcal{M}_{n}$, a field monomorphism $\sigma$ of $\mathbb{F}$, and an $\mathbb{F}^{*}$-valued mapping $\mu$ defined on the rank one matrices in $\mathcal{S}$ such that

$$
\phi(A)=\mu(A) S\left(\sigma\left(a_{i j}\right)\right) S^{-1} \text { for all rank one } A=\left(a_{i j}\right) \in \mathcal{S} .
$$


Our paper is organized as follows. In Section 2, we use a result from [19] to characterize zero product preservers. In Section 3, we discuss some immediate applications of the theorem on zero product preservers to rank preservers and mappings on $\mathbb{F}^{n}$ that preserve orthogonal pairs. In Section 4, we study mappings on $\mathcal{S}$ satisfying (1.1) for unitary similarity invariant functions $F$, including all unitarily invariant norms on real and complex matrices. A self-contained elementary proof of Theorem 2.1 (without invoking the result in [19]) is given in Section 5. It is apparent from our elementary proof that when $n=2$, we do not always have a field monomorphism $\sigma$ as in the case of $n \geq 3$. The idea of the proof may be useful in further extending the result on zero product preservers to matrices over more general rings such as division rings.

In our discussion, $\mathbb{F}^{*}$ denotes the multiplicative group of all nonzero elements in $\mathbb{F}$, $\left\{e_{1}, \ldots, e_{n}\right\}$ denotes the standard basis for $\mathbb{F}^{n}$, and $e=e_{1}+\cdots+e_{n}$. The standard basis for $\mathcal{M}_{n}$ is denoted by $\left\{E_{11}, E_{12}, \ldots, E_{n n}\right\}$, and $\mathcal{M}_{n}^{m}$ denotes the semigroup of matrices in $\mathcal{M}_{n}$ having rank at most $m$, where $m \in\{1, \ldots, n\}$.

For any matrix $A=\left(a_{i j}\right) \in \mathcal{M}_{n}$ and field homomorphism $\sigma$ of $\mathbb{F}$, denote by $A_{\sigma}$ the matrix whose $(i, j)$-th entry is $\sigma\left(a_{i j}\right)$, that is, $A_{\sigma}=\left(\sigma\left(a_{i j}\right)\right)$. Note that

(a) $A_{\sigma} B_{\sigma}=(A B)_{\sigma}$ for all $A, B \in \mathcal{M}_{n}$.

If $\sigma$ is a monomorphism, we also have

(b) $B_{\sigma}^{-1}=\left(B^{-1}\right)_{\sigma}$ for all invertible $B \in \mathcal{M}_{n}$; and

(c) $\operatorname{rank} A_{\sigma}=\operatorname{rank} A$ for all $A \in \mathcal{M}_{n}$.

\section{Zero product preserving mappings}

Motivated by theory and applications, researchers have studied the basic preserver result on linear, additive, or bijective mappings $\phi$ preserving zero products in both directions, that is,

$$
\phi(A) \phi(B)=0 \quad \text { if and only if } \quad A B=0 \text { for all } A, B \in \mathcal{S}
$$

on various subsets $\mathcal{S}$ of an algebra; see $[3,7,12,18,19]$.

In [12], Molnár studied zero product preservers on the set of all bounded linear rank one idempotent operators acting on the Banach space $\mathcal{X}$. If $\mathcal{X}$ is complex and has finite dimension $n$, then operators on $\mathcal{X}$ can be identified as $n \times n$ complex matrices. Molnár showed that if $n \geq 3$, bijective zero product preservers on the $n \times n$ rank one idempotent matrices have the form

$$
\left(a_{i j}\right) \mapsto S\left(\sigma\left(a_{i j}\right)\right) S^{-1}
$$

for some invertible matrix $S$ and field automorphism $\sigma$. 
In [19], Šemrl used the Fundamental Theorem of Projective Geometry to give a short proof of an improved version of the above result. Specifically, he considered matrices over any field and mappings preserving zero products in one direction only, which may not be bijective. These zero product preservers have the same form as above, except that $\sigma$ can now be a field monomorphism.

REMARK. Theorem 1.2 in [19] only asserts that the mapping $\phi$ has the form (2.1) for some field endomorphism $\sigma$. Nevertheless, it is clear from the proof of [19, Theorem 1.2] that $\sigma$ is nonzero, and hence is a monomorphism.

Compared with the result of Šemrl in [19], ours assumes that $\phi$ preserves zero products in both directions as a trade off for not requiring rank one idempotents be mapped to rank one idempotents. Indeed, the two conditions are more or less equivalent, as we will see that under the stronger assumption, $\phi$ maps rank one idempotents to scalar multiples of rank one idempotents.

We are not able to exhaust all those $\phi$ preserving zero products in one direction only. There are more examples than that described above. For instance, the $\phi$ that maps all matrices to scalar multiples of a fixed square-zero matrix. It is also clear that such $\phi$ may not preserve the set of rank one matrices.

The main theorem of this section is the following. An infinite dimensional version of the result under a different setting and the bijective assumption is proved in [21, Theorem 4.1].

THEOREM 2.1. Let $\mathcal{S}$ be a subset of $\mathcal{M}_{n}$ containing all rank one idempotents. Suppose $\phi: \mathcal{S} \rightarrow \mathcal{M}_{n}$ is zero product preserving, that is,

$$
\phi(A) \phi(B)=0 \text { if and only if } A B=0 \text { for all } A, B \in \mathcal{S} .
$$

Then there are functions $f, g: \mathbb{F}^{n} \rightarrow \mathbb{F}^{n}$ such that $y^{t} x=0$ if and only if $g(y)^{t} f(x)=$ 0 , and there is a $\mathbb{F}^{*}$-valued mapping $\mu$ on the rank one matrices in $\mathcal{S}$ such that

$$
\phi\left(x y^{t}\right)=\mu\left(x y^{t}\right) f(x) g(y)^{t}
$$

for all rank one matrix $x y^{t} \in \mathcal{S}$. Suppose $n \geq 3$. Then there exist an invertible $S \in$ $\mathcal{M}_{n}$, and a field monomorphism $\sigma$ of $\mathbb{F}$ such that $f(x)=S x_{\sigma}$ and $g(y)=\left(S^{-1}\right)^{t} y_{\sigma}$, that is,

$$
\phi(A)=\mu(A) S A_{\sigma} S^{-1} \quad \text { for all rank one } A \in \mathcal{S} .
$$

Furthermore, if $A=M\left(I_{k} \oplus 0_{n-k}\right) N \in \mathcal{S}$ for some invertible $M, N \in \mathcal{M}_{n}$ and $k>1$, then

$$
\phi(A)=S M_{\sigma}\left(A_{k} \oplus 0_{n-k}\right) N_{\sigma} S^{-1}
$$

for some matrix $A_{k} \in \mathcal{M}_{k}$. Consequently, if $\mathbb{F}$ has the property that all its nonzero (field) endomorphisms are automorphisms, then $A$ and $\phi(A)$ have the same rank for each $A \in \mathcal{S}$. 
It follows from Theorem 2.1 that a zero product preserving map is always rank one preserving and rank-k non-increasing for $k>1$. We are indebted to Peter Šemrl who showed us the last assertion of the theorem and the following example showing that $\phi$ may indeed decrease rank if $\mathbb{F}$ is the complex field.

Suppose $n \geq 3, \mathcal{M}_{n}$ is the set of $n \times n$ matrices over $\mathbb{C}$, and $\mathcal{S} \subseteq \mathcal{M}_{n}$ consists of all rank one idempotents and the matrix $E_{11}+E_{22}$. By the result of [9], there exist $a, b, c \in \mathbb{C}$ and a field monomorphism $\sigma$ of $\mathbb{C}$ such that $a, b, c$ are algebraically independent of $\sigma(\mathbb{C})$, that is, if $p\left(z_{1}, z_{2}, z_{3}\right)$ is a polynomial with coefficients in $\sigma(\mathbb{C})$ such that $p(a, b, c)=0$, then $p$ is the zero polynomial. Define $\phi: \mathcal{S} \rightarrow \mathcal{M}_{n}$ such that $\phi(P)=P_{\sigma}$ for all rank one idempotent $P$ and

$$
\phi\left(E_{11}+E_{22}\right)=\left(\begin{array}{cc}
a & b \\
c a & c b
\end{array}\right) \oplus 0_{n-2}
$$

Then $\phi$ is a zero product preserving map and

$$
\operatorname{rank}\left(E_{11}+E_{22}\right)=2>1=\operatorname{rank}\left(\phi\left(E_{11}+E_{22}\right)\right) .
$$

We need several lemmas to prove Theorem 2.1. The first one is a characterization of (multiples of) rank one idempotents in terms of product zero. For any $A \in \mathcal{M}_{n}$, let $N(A)=\left\{x \in \mathbb{F}^{n}: A x=0\right\}$ and $R(A)=\left\{A x: x \in \mathbb{F}^{n}\right\}$ be the null space and the column space of $A$, respectively.

Lemma 2.2. Let $A_{1}, \ldots, A_{n} \in \mathcal{M}_{n}$. Then

$$
A_{j} A_{i}=A_{i} A_{j}=0 \neq A_{j}^{2} \text { for all } i \neq j
$$

if and only if there exist an invertible $S$, and nonzero numbers $r_{1}, \ldots, r_{n}$ such that $S^{-1} A_{j} S=r_{j} E_{j j}$ for $j=1, \ldots, n$.

PROOF. Suppose $n \geq 3$ and that $A_{1}, \ldots, A_{n}$ satisfy (2.3) with one of the $A_{i}$ 's having rank greater than one. Say, $\operatorname{rank} A_{1}>1$. Then $\operatorname{dim} N\left(A_{1}\right)=n-\operatorname{rank} A_{1}<n-1$. As $A_{1} A_{j}=0, R\left(A_{j}\right) \subseteq N\left(A_{1}\right)$ for all $2 \leq j \leq n$, and hence $R\left(A_{2}\right)+\cdots+R\left(A_{n}\right) \subseteq$ $N\left(A_{1}\right)$. There must be some $j, 2<j \leq n$, such that

$$
R\left(A_{j}\right) \subseteq R\left(A_{2}\right)+\cdots+R\left(A_{j-1}\right) .
$$

Otherwise,

$$
\operatorname{dim}\left(R\left(A_{2}\right)+\cdots+R\left(A_{n}\right)\right) \geq n-1>\operatorname{dim} N\left(A_{1}\right) .
$$

We also have $R\left(A_{2}\right), \ldots, R\left(A_{j-1}\right) \subseteq N\left(A_{j}\right)$. Hence

$$
R\left(A_{j}\right) \subseteq R\left(A_{2}\right)+\cdots+R\left(A_{j-1}\right) \subseteq N\left(A_{j}\right),
$$


and $A_{j}^{2}=0$, which contradicts (2.3). So each $A_{j}$ has rank one.

It is clear that the same conclusion holds when $n=2$.

Since $A_{j}^{2} \neq 0, A_{j}$ is not a nilpotent. So, each $A_{j}$ has one nonzero eigenvalue and is similar to an upper triangular matrix with one nonzero row.

Now $A_{1}, \ldots, A_{n}$ are mutually commuting and they are simultaneously triangularizable. Take an invertible $S \in \mathcal{M}_{n}$ so that $S^{-1} A_{1} S=r_{1} E_{11}$. Then $A_{1} A_{j}=A_{j} A_{1}=0$ implies that $S^{-1} A_{j} S=[0] \oplus B_{j}$ for $j=2, \ldots, n$. Since $B_{i} B_{j}=B_{j} B_{i}=0$ for all $i \neq j$, we can use an inductive argument to show that there is an invertible $T$ of the form [1] $\oplus T_{0}$ such that $T^{-1}\left([0] \oplus B_{j}\right) T=r_{j} E_{j j}$ for $j=2, \ldots, n$. Replacing $S$ by $S T$, we have $S^{-1} A_{j} S=r_{j} E_{j j}$ for $j=1, \ldots, n$.

The converse is clear.

Now let $\mathcal{S}$ be a subset of $\mathcal{M}_{n}$ containing all rank one idempotents, and $\phi: \mathcal{S} \rightarrow \mathcal{M}_{n}$ be a mapping satisfying (2.2), that is,

$$
\phi(A) \phi(B)=0 \quad \text { if and only if } A B=0 \text { for all } A, B \in \mathcal{S} .
$$

By Lemma 2.2, $\phi$ maps rank one idempotents to scalar multiples of rank one idempotents. It is clear that the scalars can be arbitrary. It is also clear that for every $A \in \mathcal{S}$, $\phi(A)$ can only be determined up to a scalar multiple. For any $A, B \in \mathcal{M}_{n}$, we write $A \equiv B$ if $A=\lambda B$ for some nonzero $\lambda \in \mathbb{F}$. Note that

$$
A_{1} \equiv A_{2} \text { and } B_{1} \equiv B_{2} \quad \text { implies } \quad A_{1} B_{1} \equiv A_{2} B_{2} .
$$

Moreover, $A \equiv 0$ if and only if $A=0$.

LEMMA 2.3. The mapping $\phi$ maps rank one matrices to rank one matrices. Moreover, for any rank one matrices $A$ and $B$ in $\mathcal{S}$,

(a) $R(A)=R(B)$ implies $R(\phi(A))=R(\phi(B))$; and

(b) $N(A)=N(B)$ implies $N(\phi(A))=N(\phi(B))$.

Consequently, $A \equiv B$ implies $\phi(A) \equiv \phi(B)$.

PRoOF. Suppose $R(A)=R(B)$ for rank one matrices $A$ and $B$. Take a nonzero $x \in R(A)$ and form a basis $\left\{x_{1}, \ldots, x_{n}\right\}$ for $\mathbb{F}^{n}$ with $x_{1}=x$. Let $A_{i}=x_{i} y_{i}^{t}$, where $\left[y_{1}|\cdots| y_{n}\right]^{t}$ is the inverse of the matrix $\left[x_{1}|\cdots| x_{n}\right]$. Then $A_{1}, \ldots, A_{n}$ satisfy (2.3), and so do $\phi\left(A_{1}\right), \ldots, \phi\left(A_{n}\right)$. By Lemma 2.2, there is an invertible $S \in \mathcal{M}_{n}$ such that $\phi\left(A_{i}\right)=r_{i} S E_{i i} S^{-1}$ for some nonzero $r_{i}$. Since $A_{i} x=0$, we have that $A_{i} A=A_{i} B=0$ for all $i=2, \ldots, n$. By $(2.2), \phi\left(A_{i}\right) \phi(A)=\phi\left(A_{i}\right) \phi(B)=0$ for all $i=2, \ldots, n$. Hence $R(\phi(A)), R(\phi(B)) \subseteq N\left(\phi\left(A_{2}\right)\right) \cap \cdots \cap N\left(\phi\left(A_{n}\right)\right)$. As $\operatorname{dim} N\left(\phi\left(A_{2}\right)\right) \cap \cdots \cap N\left(\phi\left(A_{n}\right)\right)=1$, we see that $\phi(A)$ has rank one, and $R(\phi(A))=R(\phi(B))$. So, the first assertion and condition (a) hold. Part (b) can be obtained by a similar argument. 
The last assertion follows from the fact that for rank one matrices $A$ and $B, A \equiv B$ if and only if $R(A)=R(B)$ and $N(A)=N(B)$.

Now, we can present the proof of Theorem 2.1.

PROOF. By Lemma 2.3, we see that $\phi\left(x y^{t}\right)=\mu\left(x y^{t}\right) f(x) g(y)^{t}$ for some functions $f, g$ defined on $\mathbb{F}^{n}$ and $\mathbb{F}^{*}$-valued mapping $\mu$ on the rank one matrices in $\mathcal{S}$. Also, for any rank one matrix $x y^{t} \in \mathcal{S}, x y^{t}$ is nilpotent if and only if $\phi\left(x y^{t}\right)$ is. Hence $y^{t} x=0$ if and only if $g(y)^{t} f(x)=0$, as asserted.

Suppose $n \geq 3$. Since $\phi$ maps rank one idempotents to multiples of rank one idempotents, by [19, Theorem 1.2] (and the remark before the theorem), there exist an invertible $S \in \mathcal{M}_{n}$ and a field monomorphism $\sigma$ of $\mathbb{F}$ such that $\phi(A) \equiv S A_{\sigma} S^{-1}$ for all rank one idempotents $A \in \mathcal{S}$. In other words,

$$
\phi\left(x y^{t}\right) \equiv S\left(x y^{t}\right)_{\sigma} S^{-1}=\left(S x_{\sigma}\right)\left(\left(S^{-1}\right)^{t} y_{\sigma}\right)^{t}
$$

for all rank one matrices $x y^{t}$ such that $y^{t} x=1$. Comparing the formula to the preceding representation, we may choose $f(x)=S x_{\sigma}$ and $g(y)=\left(S^{-1}\right)^{t} y_{\sigma}$.

Now, suppose $A \in \mathcal{S}$ has the form $A=M\left(I_{k} \oplus 0_{n-k}\right) N$ for some invertible $M, N \in \mathcal{M}_{n}$ and $k>1$. The assertion is trivial if $k=n$. Suppose $k<n$. For any $j=k+1, \ldots, n$, let $B=M E_{j j} M^{-1}$ and $C=N^{-1} E_{j j} N$. Then

$$
\phi(B)=\mu(B) S B_{\sigma} S^{-1}=\mu(B) S\left(M E_{j j} M^{-1}\right)_{\sigma} S^{-1}=\mu(B) S M_{\sigma} E_{j j} M_{\sigma}^{-1} S^{-1} .
$$

Similarly,

$$
\phi(C)=\mu(C) S N_{\sigma}^{-1} E_{j j} N_{\sigma} S^{-1} .
$$

Clearly, $B A=0=A C$. Then $\phi(B) \phi(A)=0=\phi(A) \phi(C)$. It follows that

$$
\phi(A)=S M_{\sigma}\left(A_{k} \oplus 0_{n-k}\right) N_{\sigma} S^{-1}
$$

for some $A_{k} \in \mathcal{M}_{k}$.

Finally, suppose $\mathbb{F}$ has the additional property that all of its nonzero endomorphisms are automorphisms. Then we can replace $\phi$ by $A \mapsto \mu(A)^{-1} S^{-1} \phi\left(A_{\sigma^{-1}}\right) S$ and assume that $\phi(P)=P$ for all rank one idempotent $P \in \mathcal{S}$. Then for every $A \in \mathcal{S}$, we have $A P=0$ if and only if $\phi(A) P=0$, and $P A=0$ if and only if $P \phi(A)=0$, for all rank one idempotent $P$. Thus, $\phi(A)$ and $A$ have the same image and kernel. So, $A$ and $\phi(A)$ have the same rank. The proof of the theorem is complete.

\section{Rank preservers on $\mathcal{M}_{n}^{m}$ and orthogonality preservers on $\mathbb{F}^{n}$}

As an immediate application of Theorem 2.1, we characterize rank preserving mappings on the semigroup $\mathcal{M}_{n}^{m}$ of $\mathcal{M}_{n}$, that is, $\phi: \mathcal{M}_{n}^{m} \rightarrow \mathcal{M}_{n}$ such that

$$
\operatorname{rank}(\phi(A) \phi(B))=\operatorname{rank}(A B) \text { for all } A, B \in \mathcal{M}_{n}^{m} \text {. }
$$


Clearly such a $\phi$ satisfies (2.2). When $m=n=2$, one can apply Theorem 2.1 to gain some information about $\phi$ on rank one matrices. However, $\phi$ can map the set of invertible matrices into itself in any way we like. We consider $n \geq 3$ in the following.

THEOREM 3.1. Suppose $n \geq 3$. A mapping $\phi: \mathcal{M}_{n}^{m} \rightarrow \mathcal{M}_{n}$ satisfying (3.1) has

$$
\operatorname{rank}(\phi(A) \phi(B))=\operatorname{rank}(A B) \text { for all } A, B \in \mathcal{M}_{n}^{m}
$$

if and only if there exist an invertible $S \in \mathcal{M}_{n}$, and a field monomorphism $\sigma$ of $\mathbb{F}$ such that for any $A=M\left(I_{k} \oplus 0_{n-k}\right) N \in \mathcal{M}_{n}^{m}$ with invertible $M, N$,

$$
\phi(A)=S M_{\sigma}\left(A_{k} \oplus 0_{n-k}\right) N_{\sigma} S^{-1}
$$

for some invertible matrix $A_{k} \in \mathcal{M}_{k}$.

Proof. Suppose $\phi$ has the desired form. For any $A=M\left(I_{k} \oplus 0_{n-k}\right) N \in \mathcal{M}_{n}^{m}$ with $M, N$ invertible,

$$
\phi(A)=S M_{\sigma}\left(A_{k} \oplus 0_{n-k}\right) N_{\sigma} S^{-1}=S P_{A} A_{\sigma} S^{-1}=S A_{\sigma} Q_{A} S^{-1}
$$

for $P_{A}=M_{\sigma}\left(A_{k} \oplus I_{n-k}\right) M_{\sigma}^{-1}$ and $Q_{A}=N_{\sigma}^{-1}\left(A_{k} \oplus I_{n-k}\right) N_{\sigma}$. Then for any $A, B \in \mathcal{M}_{n}^{m}$,

$$
\phi(A) \phi(B)=S P_{A} A_{\sigma} B_{\sigma} Q_{B} S^{-1}=S P_{A}(A B)_{\sigma} Q_{B} S^{-1} .
$$

Since $S, P_{A}$, and $Q_{B}$ are invertible, $\operatorname{rank}(\phi(A) \phi(B))=\operatorname{rank}(A B)_{\sigma}=\operatorname{rank}(A B)$. The sufficiency part holds.

For necessity, note that $\phi$ must also satisfy (2.2). By Theorem 2.1, there exist an invertible $S \in \mathcal{M}_{n}$ and a field monomorphism $\sigma$ of $\mathbb{F}$ such that for any $A=$ $M\left(I_{k} \oplus 0_{n-k}\right) N \in \mathcal{M}_{n}^{m}$ with invertible $M, N$,

$$
\phi(A)=S M_{\sigma}\left(A_{k} \oplus 0_{n-k}\right) N_{\sigma} S^{-1}
$$

for some $A_{k} \in \mathcal{M}_{k}$. We need to show $A_{k}$ is invertible. Let $B=N^{-1}\left(I_{k} \oplus 0_{n-k}\right) \in \mathcal{M}_{n}^{m}$. Then $A B=M\left(I_{k} \oplus 0_{n-k}\right)$ has rank $k$. Hence

$$
\begin{aligned}
\phi(A) \phi(B) & =S M_{\sigma}\left(A_{k} \oplus 0_{n-k}\right) N_{\sigma} S^{-1} S N_{\sigma}^{-1}\left(B_{k} \oplus 0_{n-k}\right) S^{-1} \\
& =S M_{\sigma}\left(A_{k} B_{k} \oplus 0_{n-k}\right) S^{-1}
\end{aligned}
$$

has rank $k$. It follows that $A_{k}$ and $B_{k}$ are invertible.

Next we show that our main theorem can be used to study mappings on $\mathbb{F}^{n}$ preserving orthogonality; see [1]. We write $u \equiv v$ if $u$ is a scalar multiple of $v$. 
Proposition 3.2. Let $n \geq 3$ and let $f: \mathbb{F}^{n} \rightarrow \mathbb{F}^{n}$ be a mapping such that $x^{t} y=0$ if and only if $f(x)^{t} f(y)=0$ for every $x, y \in \mathbb{F}^{n}$. Then there exist $S \in \mathcal{M}_{n}$ with $S^{\prime} S=I_{n}$, and a field monomorphism $\sigma$ of $\mathbb{F}$ such that $f(x) \equiv S x_{\sigma}$ for all $x \in \mathbb{F}^{n}$.

Instead of proving this proposition, we present the result and proof for a slightly more involved version for the inner product $(x, y)=y^{*} x$ on $\mathbb{C}^{n}$.

PROPOSITION 3.3. Let $n \geq 3$ and let $f: \mathbb{C}^{n} \rightarrow \mathbb{C}^{n}$ be a mapping such that $x^{*} y=0$ if and only if $f(x)^{*} f(y)=0$ for every $x, y \in \mathbb{C}^{n}$. Then there exist a unitary $S \in \mathcal{M}_{n}$ and $\sigma: \mathbb{C} \rightarrow \mathbb{C}$ of the form $z \mapsto z$ or $z \mapsto \bar{z}$ such that $f(x) \equiv S x_{\sigma}$ for all $x \in \mathbb{C}^{n}$.

PROOF. For every nonzero rank one matrix $A \in \mathcal{M}_{n}$, write $A=x y^{*}$ and define $\phi(A)=f(x) f(y)^{*}$. Also let $\phi(0)=0$. Then it is easy to check that $\phi: \mathcal{M}_{n}^{1} \rightarrow \mathcal{M}_{n}$ is zero product preserving.

By Theorem 2.1, there exist an invertible $S \in \mathcal{M}_{n}$, a field monomorphism $\sigma$ of $\mathbb{C}$ and a mapping $\mu: \mathcal{M}_{n}^{1} \backslash\{0\} \rightarrow \mathbb{C}^{*}$ such that for all nonzero $x, y \in \mathbb{C}^{n}$,

$$
f(x) f(y)^{*}=\phi\left(x y^{*}\right)=\mu\left(x y^{*}\right) S\left(x y^{*}\right)_{\sigma} S^{-1}=\mu\left(x y^{*}\right)\left(S x_{\sigma}\right)\left(\left(y^{*}\right)_{\sigma} S^{-1}\right) .
$$

We conclude that $f(x) \equiv S x_{\sigma}$ and $f(y) \equiv\left(S^{-1}\right)^{*}\left(\left(y^{*}\right)_{\sigma}\right)^{*}$. Setting $x=y$, we get $S x_{\sigma} \equiv\left(S^{-1}\right)^{*}\left(\left(x^{*}\right)_{\sigma}\right)^{*}$, and hence $\left(S^{*} S\right) x_{\sigma} \equiv\left(\left(x^{*}\right)_{\sigma}\right)^{*}$.

For $x=e_{j}$, we have $\left(S^{*} S\right) e_{j} \equiv e_{j}$. All $e_{j}$ are eigenvectors of $S^{*} S$ and hence $S^{*} S$ is a diagonal matrix. However, we also have $\left(S^{*} S\right) e \equiv e$. The diagonal matrix $S^{*} S$ is indeed a scalar matrix. Absorbing the scalar into the function $\mu$, we assume that $S^{*} S$ is the identity matrix, or equivalently, that $S$ is unitary.

Now $x_{\sigma} \equiv\left(\left(x^{*}\right)_{\sigma}\right)^{*}$ for every $x \in \mathbb{C}^{n}$. For every $\lambda \in \mathbb{C}$, let $x=(\lambda, 1,0, \ldots, 0)^{t}$. Since

$$
(\sigma(\lambda), 1,0, \ldots, 0)^{t}=x_{\sigma} \equiv\left(\left(x^{*}\right)_{\sigma}\right)^{*}=(\overline{\sigma(\bar{\lambda})}, 1,0, \ldots, 0)^{t},
$$

where $\overline{\sigma(\lambda)}=\sigma(\bar{\lambda})$. However, then $\overline{\sigma(\lambda)}=\sigma(\lambda)$ for every $\lambda \in \mathbb{R}$ so that $\sigma$ maps $\mathbb{R}$ into $\mathbb{R}$. It follows that $\sigma$ has the form $z \mapsto z$ or $z \mapsto \bar{z}$; see [22].

Peter Šemrl pointed out that the above proposition is the non-bijective finitedimensional version of Uhlhorn's theorem in quantum mechanics; see [17] and also [12].

\section{Unitarily invariant and unitary similarity invariant functions}

In this section, $\mathcal{S}$ denotes a subset of $\mathcal{M}_{n}$ containing all rank one matrices. Also, we focus on the case when $\mathbb{F}=\mathbb{R}$ or $\mathbb{C}$. Let $\mathcal{U}_{n}=\left\{U \in \mathcal{M}_{n}: U^{*} U=I_{n}\right\}$. We study $\phi: \mathcal{S} \rightarrow \mathcal{M}_{n}$ such that

$$
F(\phi(A) \phi(B))=F(A B) \text { for all } A, B \in \mathcal{S},
$$


where $F: \mathcal{M}_{n} \rightarrow[0, \infty)$ satisfies the following conditions:

(F1) $F(A)=0$ if and only if $A=0$.

(F2) There is a $p \in \mathbb{R}^{*}$ such that $F(\lambda A)=|\lambda|^{p} F(A)$ for all $\lambda \in \mathbb{F}^{*}$.

(F3) $F(U A V)=F(A)$ for all $U, V \in \mathcal{U}_{n}$.

This class of functions includes all common matrix norms on $\mathcal{M}_{n}$ such as the spectral norm $\|A\|=\max \left\{\|A x\|: x \in \mathbb{F}^{n},\|x\| \leq 1\right\}$, and the Frobenius norm $\|A\|_{F}=\operatorname{tr}\left(A^{*} A\right)^{1 / 2}$. However, the triangle inequality is not assumed.

We may always assume that $p=1$ in (F2). Otherwise, we can replace $F$ by the mapping $A \mapsto|F(A)|^{1 / p}$. A function $F$ satisfying (F3) is known as a unitarily invariant function. Evidently, condition (4.1) will still hold after this replacement. We will always assume this in our discussion.

We have the following result.

THEOREM 4.1. Let $F: \mathcal{M}_{n} \rightarrow[0, \infty)$ satisfy $(\mathrm{F} 1)-(\mathrm{F} 3)$. Suppose $n \geq 3$, and $\phi: \mathcal{S} \rightarrow \mathcal{M}_{n}$ is a mapping satisfying

$$
F(\phi(A) \phi(B))=F(A B) \text { for all } A, B \in \mathcal{S} \text {. }
$$

Then there exist a matrix $W \in \mathcal{U}_{n}$, and mappings $\psi_{L}, \psi_{R}: \mathcal{S} \rightarrow \mathcal{U}_{n}$ satisfying

$$
\psi_{L}(A) A=A \psi_{R}(A) \text { for all } A \in \mathcal{S}
$$

such that one of the following holds:

(a1) $F\left(\psi_{L}(A) A B \psi_{R}(B)\right)=F(A B)$ for all $A, B \in \mathcal{S}$, and $\phi$ has the form

$$
A \mapsto W \psi_{L}(A) A W^{*}=W A \psi_{R}(A) W^{*} .
$$

(a2) $\mathbb{F}=\mathbb{C}, F\left(\overline{\psi_{L}(A) A B \psi_{R}(B)}\right)=F(A B)$ for all $A, B \in \mathcal{S}$, and $\phi$ has the form

$$
A \mapsto W \overline{\psi_{L}(A) A} W^{*}=W \overline{A \psi_{R}(A)} W^{*} .
$$

Suppose $A=X D Y$, where $X, Y \in \mathcal{U}_{n}$ and $D=\operatorname{diag}\left(s_{1}(A), \ldots, s_{n}(A)\right)$ for singular values $s_{1}(A), \ldots, s_{n}(A)$ of $A$. Then $\psi_{L}(A) A=A \psi_{R}(A)$ if and only if $X^{*} \psi_{L}(A) X D=D Y \psi_{R}(A) Y^{*}$. So, each of the matrices $X^{*} \psi_{L}(A) X$ and $Y \psi_{R}(A) Y^{*}$ is a direct sum of square blocks according to the multiplicities of the singular values of $A$. Moreover, the blocks corresponding to the nonzero singular values are the same in the two matrices.

It turns out that we can strengthen Theorem 4.1 by replacing assumption (F3) by the following weaker condition on $F: \mathcal{M}_{n} \rightarrow[0, \infty)$.

(F3') $F\left(U^{*} A U\right)=F(A)$ for all $U \in \mathcal{U}_{n}$. 
A function $F$ satisfying $\left(\mathrm{F}^{\prime}\right)$ is called a unitary similarity invariant function. Clearly, a unitarily invariant function is unitary similarity invariant. The following result shows that preservers of unitary similarity invariant functions have the same structure of preservers of unitarily invariant functions, and more can be said if $F$ is not unitarily invariant on rank one matrices.

THEOREM 4.2. Let $F: \mathcal{M}_{n} \rightarrow[0, \infty)$ satisfy $(\mathrm{F} 1),(\mathrm{F} 2)$ and $\left(\mathrm{F}^{\prime}\right)$. Suppose $n \geq 3$, and $\phi: \mathcal{S} \rightarrow \mathcal{M}_{n}$ is a mapping satisfying $F(\phi(A) \phi(B))=F(A B)$ for all $A, B \in \mathcal{S}$. Then condition (a1) or (a2) of Theorem 4.1 holds. Moreover, if

$$
\left\{\left|F(X) / s_{1}(X)\right|: X \in \mathcal{M}_{n}^{1}\right\}
$$

is not a singleton, then there is a mapping $\mu: \mathcal{S} \rightarrow \Pi=\{z \in \mathbb{E}:|z|=1\}$ such that $\psi_{L}(A)=\psi_{R}(A)=\mu(A) I$, and thus one of the following holds.

(b1) $\phi$ has the form $A \mapsto \mu(A) W A W^{*}$.

(b2) $\mathbb{F}=\mathbb{C}, F(\overline{A B})=F(A B)$ for all $A, B \in \mathcal{S}$, and $\phi$ has the form $A \mapsto$ $\mu(A) W \bar{A} W^{*}$.

We need only prove Theorem 4.2, and Theorem 4.1 will then follow. We begin with a condition under which $F$ is essentially the largest singular value on rank one matrices. Denote by $s_{1}(A) \geq \cdots \geq s_{n}(A)$ the singular values of $A$.

LEMMA 4.3. Suppose $n \geq 3$. If there is a vector $b \in \mathbb{F}^{n}$, which is not a multiple of $e_{1}$, such that $F\left(e_{1} y^{*}\right)=F\left(b y^{*}\right)$ for all $y \in \mathbb{F}^{n}$, then

$$
F(X)=s_{1}(X) F\left(E_{11}\right) \text { for all } X \in \mathcal{M}_{n}^{1} .
$$

PROOF. Since $b$ is not a multiple of $e_{1}$, there exists $U=[\lambda] \oplus U_{1} \in \mathcal{U}_{n}$ with $|\lambda|=1$ such that $U b=c=\left(c_{1}, c_{2}, 0, \ldots, 0\right)$ with $c_{1} \geq 0$ and $c_{2}>0$. As

$$
\begin{aligned}
F\left(c y^{*}\right) & =F\left(U^{*} c y^{*} U\right)=F\left(b y^{*} U\right)=F\left(e_{1} y^{*} U\right) \\
& =F\left(U e_{1} y^{*}\right)=F\left(\lambda e_{1} y^{*}\right)=F\left(e_{1} y^{*}\right),
\end{aligned}
$$

we may assume that $b=\left(b_{1}, b_{2}, 0, \ldots, 0\right)$ with $b_{1} \geq 0$ and $b_{2}>0$.

Let $t \in[0, \pi / 2]$. There exists a $\mu_{t}>0$ such that for any $\hat{t} \in\left(t-\mu_{t}, t+\mu_{t}\right) \cap$ $[0, \pi / 2]$, there are vectors $y=\left(\sin t, y_{2}, \ldots, y_{n}\right)$ and $z=\left(\sin \hat{t}, z_{2}, \ldots, z_{n}\right)$ with $\|y\|=\|z\|=1$ such that $\left|y^{*} b\right|=\left|z^{*} b\right|$. This is possible as $n \geq 3$.

Now the rank one matrix $e_{1} y^{*}$ is unitarily similar to $\sin t E_{11}+\cos t E_{12}$, and $b y^{*}$ is unitarily similar to a unit multiple of

$$
\left|y^{*} b\right| E_{11}+\sqrt{\left\|b y^{*}\right\|^{2}-\left|y^{*} b\right|^{2}} E_{12}=\left|y^{*} b\right| E_{11}+\sqrt{\|b\|^{2}-\left|y^{*} b\right|^{2}} E_{12} .
$$


Hence

$$
\begin{aligned}
F\left(\sin t E_{11}+\cos t E_{12}\right) & =F\left(e_{1} y^{*}\right)=F\left(b y^{*}\right) \\
& =F\left(\left|y^{*} b\right| E_{11}+\sqrt{\|b\|^{2}-\left|y^{*} b\right|^{2}} E_{12}\right) .
\end{aligned}
$$

Similarly,

$$
F\left(\sin \hat{t} E_{11}+\cos \hat{t} E_{12}\right)=F\left(\left|z^{*} b\right| E_{11}+\sqrt{\|b\|^{2}-\left|z^{*} b\right|^{2}} E_{12}\right) .
$$

As $\left|y^{*} b\right|=\left|z^{*} b\right|, F\left(\sin t E_{11}+\cos t E_{12}\right)=F\left(\sin \hat{t} E_{11}+\cos \hat{t} E_{12}\right)$.

It follows from the compactness of $[0, \pi / 2]$ that $F\left(\sin t E_{11}+\cos t E_{12}\right)=F\left(E_{11}\right)$ for all $t \in[0, \pi / 2]$. Thus, $F(X)=s_{1}(X) F\left(E_{11}\right)$ for all $X \in \mathcal{M}_{n}^{1}$.

LEMMA 4.4. Suppose $n \geq 3$. If there are matrices $A, B \in \mathcal{M}_{n}$ with $B$ not a multiple of $A$ such that $F(A X)=F(B X)$ for all $X \in \mathcal{M}_{n}^{1}$, then $F$ satisfies (4.3).

PROOF. Using Lemma 4.3, it is not difficult to see that (4.3) holds if there are vectors $b, c$ in $\mathbb{F}^{n}$, with one not a multiple of the other, such that $F\left(b y^{*}\right)=F\left(c y^{*}\right)$ for all $y \in \mathbb{F}^{n}$.

If $B$ is not a multiple of $A$, then there is an $x$ in $\mathbb{F}^{n}$ such that $B x$ is not a multiple of $A x$. However, we have $F\left(A x y^{*}\right)=F\left(B x y^{*}\right)$ for all $y \in \mathbb{F}^{n}$. The conclusion follows.

LEMMA 4.5. Suppose $F$ satisfies (4.3). Then for any matrices $A, B \in \mathcal{M}_{n}$,

$$
F(A X)=F(B X) \text { for all } X \in \mathcal{M}_{n}^{1}
$$

if and only if there is a $U \in \mathcal{U}_{n}$ such that $B=U A$. Similarly,

$$
F(Y A)=F(Y B) \text { for all } Y \in \mathcal{M}_{n}^{1}
$$

if and only if there is $a V \in \mathcal{U}_{n}$ such that $B=A V$.

ProOF. Since $F$ satisfies (4.3), $s_{1}(A X)=s_{1}(B X)$ for all $X \in \mathcal{M}_{n}^{1}$. For every nonzero $x$ in $\mathbb{F}^{n},\|A x\|=s_{1}\left(A x e_{1}^{*}\right)=s_{1}\left(B x e_{1}^{*}\right)=\|B x\|$. Hence there is a $U \in \mathcal{U}_{n}$ such that $B=U A$.

The other assertion can be obtained similarly.

Now we are ready to give the proof of Theorem 4.2.

Proof. In view of the comment before Theorem 4.1, we may assume that $p=1$ in (F2). Clearly, if $\phi$ satisfies (4.2), then it also satisfies (2.2). By Theorem 2.1, there exist an invertible $S \in \mathcal{M}_{n}$, a field monomorphism $\sigma$ of $\mathbb{F}$, and a $\mathbb{F}^{*}$-valued 
mapping $\mu$ on the rank one matrices in $\mathcal{S}$ such that $\phi(A)=\mu(A) S A_{\sigma} S^{-1}$ for any rank one matrix $A$ in $\mathcal{S}$.

We claim that $|\sigma(\alpha)|=|\alpha|$ for every $\alpha \in \mathbb{F}$. For any $\alpha \in \mathbb{F}$, take $A=E_{11}+\alpha E_{1 n}$. Then $\phi(A)=\mu(A) S\left(E_{11}+\sigma(\alpha) E_{1 n}\right) S^{-1}$. For any $1 \leq k \leq n$,

$$
\left|\mu(A) \mu\left(E_{1 k}\right)\right| F\left(S E_{1 k} S^{-1}\right)=F\left(\phi(A) \phi\left(E_{1 k}\right)\right)=F\left(A E_{1 k}\right)=F\left(E_{1 k}\right)
$$

and

$$
\left|\sigma(\alpha) \mu(A) \mu\left(E_{n k}\right)\right| F\left(S E_{1 k} S^{-1}\right)=F\left(\phi(A) \phi\left(E_{n k}\right)\right)=F\left(A E_{n k}\right)=|\alpha| F\left(E_{1 k}\right) .
$$

It follows that $|\sigma(\alpha)|=\left|\mu\left(E_{1 k}\right) / \mu\left(E_{n k}\right)\right||\alpha|$. Since $\sigma(1)=1$, we have $\left|\mu\left(E_{1 k}\right)\right|=$ $\left|\mu\left(E_{n k}\right)\right|$, and hence $|\sigma(\alpha)|=|\alpha|$ for all $\alpha \in \mathbb{F}$. It is well known that $\sigma$ must either be the identity or the complex conjugation if $\mathbb{F}=\mathbb{C}$; see, for example, [22]. Replacing $\phi$ by $A \mapsto \phi(\bar{A})$, if necessary, we may assume that $\sigma$ is the identity on $\mathbb{F}$.

By the singular value decomposition, $S=U D V$ for some $U, V \in \mathcal{U}_{n}$ and diagonal matrix $D=\operatorname{diag}\left(d_{1}, \ldots, d_{n}\right)$, where $d_{1} \geq \cdots \geq d_{n}>0$. Replacing $\phi$ by $A \mapsto U^{*} \phi\left(V^{*} A V\right) U$, we assume that $S$ is the diagonal matrix $D$.

Actually, $D$ is a scalar matrix. Note that $\phi\left(E_{k k}\right)=\mu\left(E_{k k}\right) E_{k k}$ for all $k=1, \ldots, n$. Hence

$$
\left|\mu\left(E_{k k}\right)\right|^{2} F\left(E_{k k}\right)=F\left(\phi\left(E_{k k}\right)^{2}\right)=F\left(E_{k k}^{2}\right)=F\left(E_{k k}\right),
$$

and $\left|\mu\left(E_{k k}\right)\right|=1$. Since $\phi\left(E_{11}+E_{1 n}\right)=\mu\left(E_{11}+E_{1 n}\right)\left(E_{11}+d_{1} d_{n}^{-1} E_{1 n}\right)$, we have

$$
\begin{aligned}
\left|\mu\left(E_{11}+E_{1 n}\right) \mu\left(E_{11}\right)\right| F\left(E_{11}\right) & =F\left(\phi\left(E_{11}+E_{1 n}\right) \phi\left(E_{11}\right)\right) \\
& =F\left(\left(E_{11}+E_{1 n}\right) E_{11}\right)=F\left(E_{11}\right)
\end{aligned}
$$

and

$$
\begin{aligned}
d_{1} d_{n}^{-1}\left|\mu\left(E_{11}+E_{1 n}\right) \mu\left(E_{n n}\right)\right| F\left(E_{1 n}\right) & =F\left(\phi\left(E_{11}+E_{1 n}\right) \phi\left(E_{n n}\right)\right) \\
& =F\left(\left(E_{11}+E_{1 n}\right) E_{n n}\right)=F\left(E_{1 n}\right) .
\end{aligned}
$$

We have $\left|\mu\left(E_{11}+E_{1 n}\right) \mu\left(E_{11}\right)\right|=1$, and $d_{1} d_{n}^{-1}\left|\mu\left(E_{11}+E_{1 n}\right) \mu\left(E_{n n}\right)\right|=1$. It follows that $d_{1}=d_{n}$, and that $D$ is a scalar matrix. In conclusion, we have $\phi(X)=\mu(X) X$ for any rank one matrix $X$ in $\mathcal{S}$.

We now show that $|\mu(X)|=1$ for any rank one matrix $X$. Take any rank one matrix $X$. If $X^{2} \neq 0$, then $|\mu(X)|^{2} F\left(X^{2}\right)=F\left(\phi(X)^{2}\right)=F\left(X^{2}\right)$, and hence $|\mu(X)|=1$. If $X^{2}=0$, the there exists a rank one matrix $Y$ such that both $X Y$ and $Y^{2}$ are nonzero. We have

$$
|\mu(X)| F(X Y)=|\mu(X) \mu(Y)| F(X Y)=F(\phi(X) \phi(Y))=F(X Y) .
$$

Hence $|\mu(X)|=1$ also. 
Finally, for any matrix $A \in \mathcal{S}$,

$$
\begin{aligned}
& F(\phi(A) X)=F(\phi(A) \phi(X))=F(A X) \quad \text { and } \\
& F(X \phi(A))=F(\phi(X) \phi(A))=F(X A)
\end{aligned}
$$

for any rank one matrix $X$, as $|\mu(X)|=1$. By Lemma 4.4, either

(i) $\phi(A)$ is some unit multiple of $A$ for all $A \in \mathcal{S}$; or

(ii) $F(X)=s_{1}(X) F\left(E_{11}\right)$ for any rank one matrix $X$.

If (i) holds, $\mu$ can be extended to a $\mu: \mathcal{S} \rightarrow \Pi$ such that $\phi(A)=\mu(A) A$ for all $A \in \mathcal{S}$. If (ii) holds, then by Lemma 4.5 , there exist unitary matrices $U_{A}, V_{A} \in \mathcal{U}_{n}$ such that $\phi(A)=U_{A} A=A V_{A}$. Define mappings $\psi_{L}, \psi_{R}: \mathcal{S} \rightarrow \mathcal{U}_{n}$ by $\psi_{L}(A)=U_{A}$ and $\psi_{R}(A)=V_{A}$. Then $\phi(A)=\psi_{L}(A) A=A \psi_{R}(A)$, and the result follows.

As mentioned before, Theorem 4.1 covers many functions such as all unitarily invariant norms on $\mathcal{M}_{n}$ including the spectral norm $\|A\|$ and the Frobenius norm $\|A\|_{F}=\operatorname{tr}\left(A^{*} A\right)^{1 / 2}$. Next, we consider unitary similarity invariant functions $F$ so that condition (b1) and/or (b2) of Theorem 4.2 hold. For $\mathbb{F}=\mathbb{C}$, define the numerical range of $A$ by $W(A)=\left\{x^{*} A x: x \in \mathbb{C}^{n}, x^{*} x=1\right\}$ and the numerical radius of $A$ by $r(A)=\max \{|\mu|: \mu \in W(A)\}$.

It is known and easy to check that $r(A)=0$ if and only if $A=0$, and that $r\left(U^{*} A U\right)=r(A)=r(\bar{A})$ for all $A \in \mathcal{M}_{n}$. Note that $r\left(E_{11}\right)=1 \neq 1 / 2=r\left(E_{12}\right)$. So, if $n \geq 3$, and $\phi: \mathcal{S} \rightarrow \mathcal{M}_{n}$ is a mapping satisfying (4.2) with $F(A)=r(A)$, then condition (b1) or (b2) of Theorem 4.2 holds.

Now, if $n \geq 3$, and $\phi: \mathcal{S} \rightarrow \mathcal{M}_{n}$ is a mapping satisfying (4.2) with $F(A)=W(A)$, then $r(\phi(A) \phi(B))=r(A B)$ for all $A, B \in \mathcal{M}_{n}$. Furthermore, if $A=i E_{11}$, then

$$
W(A)=\{i t: t \in[0,1]\} \neq\{-i t: t \in[0,1]\}=W(\bar{A}) .
$$

We see that $\phi$ satisfies (b1) of Theorem 4.2 only.

More generally, let $C \in \mathcal{M}_{n}$. The $C$-numerical range of $A \in \mathcal{M}_{n}$ is defined by $W_{C}(A)=\left\{\operatorname{tr}\left(C U^{*} A U\right): U \in \mathcal{U}_{n}\right\}$ and the $C$-numerical radius of $A$ is defined by $r_{C}(A)=\max \left\{|\mu|: \mu \in W_{C}(A)\right\}$. When $C=E_{11}$, these reduce to $W(A)$ and $r(A)$. We refer to [10] for some general background on the $C$-numerical range and $C$-numerical radius. If $C$ is a non-scalar matrix with nonzero trace, then (F1)-(F3) hold for $F(A)=r_{C}(A)$. If $C$ is positive semi-definite, then we can extend the analysis on $r(A)$, and $W(A)$ in the preceding paragraphs to $r_{C}(A)$, and $W_{C}(A)$ and get the same conclusion on $\phi$. We have the following result.

COROLlaRY 4.6. Let $\mathbb{F}=\mathbb{C}$ and $C \in \mathcal{M}_{n}$ be a non-scalar positive semi-definite matrix, where $n \geq 3$. Suppose $\phi: \mathcal{S} \rightarrow \mathcal{M}_{n}$ is a mapping satisfying (4.2) for $F(A)=r_{C}(A)$ or $W_{C}(A)$. Then condition (b1) or (b2) of Theorem 4.2 holds if $F(A)=r_{C}(A) ;$ and condition (b1) of Theorem 4.2 holds if $F(A)=W_{C}(A)$. 
Continue to assume $\mathbb{F}=\mathbb{C}$. A norm $v$ on $\mathcal{M}_{n}$ is unitary similarity invariant or weakly unitarily invariant if $v\left(U^{*} A U\right)=v(A)$ for all $U \in \mathcal{U}_{n}$ and $A \in \mathcal{M}_{n}$. It is known (for example, see [10]) that for every unitary similarity invariant norm $\nu$, there is a compact subset $\mathcal{K}$ of $\mathcal{M}_{n}$ such that $\nu(A)=\max \left\{r_{C}(A): C \in \mathcal{K}\right\}$. So, the $C$-numerical radius can be viewed as a building block for unitary similarity invariant norms. It would be interesting to extend Corollary 4.6 to general unitary similarity invariant norms that are not unitarily invariant on $\mathcal{S}$.

\section{A self-contained elementary proof for Theorem 2.1}

In this section, we give a self-contained elementary proof of the second part of Theorem 2.1. The proof continues from Lemma 2.3. Replacing $\phi$ by $A \mapsto S^{-1} \phi(A) S$ for some suitable $S \in \mathcal{M}_{n}$, we may assume that

$$
\phi\left(E_{j j}\right)=r_{j} E_{j j} \quad \text { for all } 1 \leq j \leq n .
$$

LEMMA 5.1. For any rank one matrix $A$, the $(i, j)$-th entry of $\phi(A)$ is zero if and only if that of $A$ is.

PROOF. Suppose $a_{i j}$, the $(i, j)$-th entry of the matrix $A$, is 0 . Then since $A$ has rank one, either the $i$-th row, or the $j$-th column, of $A$ is zero. Respectively, $E_{i i} A=0$ or $A E_{j j}=0$. Because of (5.1), either $r_{i i} E_{i i} \phi(A)=0$, or $\phi(A) r_{j} E_{j j}=0$. In both cases, the $(i, j)$-th entry of $\phi(A)$ is zero.

The converse is similar.

LEMMA 5.2. Replacing $\phi$ by the mapping $A \mapsto D^{-1} \phi(A) D$ for some invertible diagonal $D \in \mathcal{M}_{n}$, we may further assume that

$$
\phi\left(e e^{t}\right)=e y^{t}
$$

for some $y=\left(y_{1}, \ldots, y_{n}\right)^{t}$ with all $y_{i}$ nonzero.

ProOF. Since $\phi$ maps rank one matrices to rank one matrices, $\phi\left(e e^{t}\right)=x z^{t}$ for some $x=\left(x_{1}, \ldots, x_{n}\right)^{t}, z=\left(z_{1}, \ldots, z_{n}\right)^{t} \in \mathbb{F}^{n}$. By Lemma 5.1, all entries of $\phi\left(e e^{t}\right)$ are nonzero, and hence all $x_{i}$ and $z_{i}$ are nonzero. Let $D=\operatorname{diag}\left(x_{1}, \ldots, x_{n}\right)$. Then $x=D e$, and $D^{-1} \phi\left(e e^{t}\right) D=D^{-1} x z^{t} D=e z^{t} D$. Replacing $\phi$ by $A \mapsto D^{-1} \phi(A) D$, the new mapping satisfies (5.2) with $y=D^{t} z$.

Lemma 5.3. Suppose $\phi: \mathcal{S} \rightarrow \mathcal{M}_{n}$ satisfies (2.2) and (5.1)-(5.2). When $n \geq 3$, there exists a field monomorphism $\sigma$ of $\mathbb{F}$ such that $\phi(A) \equiv A_{\sigma}$ for all rank one matrices $A$ in $\mathcal{S}$ with nonzero trace. 
ProOF. We claim that for each $j \in\{1, \ldots, n\}$, there exists a monomorphism $\sigma_{j}$ of F such that

$$
\phi(A) \equiv A_{\sigma_{j}} \quad \text { for all rank one } A \in \mathcal{S} \text { with } a_{j j} \neq 0 .
$$

Suppose (5.3) is proved. Then for any $i, j \in\{1, \ldots, n\}$ with $i \neq j$, and any nonzero $\alpha \in \mathbb{F}$,

$$
\begin{aligned}
E_{i i}+ & E_{j j}+\sigma_{i}(\alpha) E_{i j}+\sigma_{i}\left(\alpha^{-1}\right) E_{j i} \\
& \equiv \sigma_{i}(1) E_{i i}+\sigma_{i}(1) E_{j j}+\sigma_{i}(\alpha) E_{i j}+\sigma_{i}\left(\alpha^{-1}\right) E_{j i} \\
& \equiv \phi\left(E_{i i}+E_{j j}+\alpha E_{i j}+\alpha^{-1} E_{j i}\right) \\
& \equiv \sigma_{j}(1) E_{i i}+\sigma_{j}(1) E_{j j}+\sigma_{j}(\alpha) E_{i j}+\sigma_{j}\left(\alpha^{-1}\right) E_{j i} \\
& \equiv E_{i i}+E_{j j}+\sigma_{j}(\alpha) E_{i j}+\sigma_{j}\left(\alpha^{-1}\right) E_{j i} .
\end{aligned}
$$

Thus $\sigma_{i}(\alpha)=\sigma_{j}(\alpha)$. Choosing $\sigma$ to be the common monomorphism, the conclusion follows.

We prove (5.3) by a sequence of assertions. Assume that $j=1$ for simplicity.

ASSERTION 1. There exist injective mappings $f_{2}, \ldots, f_{n}, g_{2}, \ldots, g_{n}$ on $\mathbb{F}$ such that for all $x_{2}, \ldots, x_{n}, y_{2}, \ldots, y_{n} \in \mathbb{F}$,

$$
\phi\left(( \begin{array} { c } 
{ 1 } \\
{ x _ { 2 } } \\
{ \vdots } \\
{ x _ { n } }
\end{array} ) ( \begin{array} { l l l l } 
{ 1 } & { y _ { 2 } } & { \cdots } & { y _ { n } ) }
\end{array} ) \equiv ( \begin{array} { c } 
{ 1 } \\
{ f _ { 2 } ( x _ { 2 } ) } \\
{ \vdots } \\
{ f _ { n } ( x _ { n } ) }
\end{array} ) \left(\begin{array}{llll}
1 & g_{2}\left(y_{2}\right) & \cdots & \left.g_{n}\left(y_{n}\right)\right) .
\end{array}\right.\right.
$$

Furthermore, $f_{j}(1)=1$ and $g_{j}(\alpha)=-f_{j}\left(-\alpha^{-1}\right)^{-1}$ for all nonzero $\alpha \in \mathbb{F}$.

PROOF. For each $j>1$ and $\alpha \neq 0$, we have, by Lemma 5.1, nonzero $\beta_{\alpha}$ and $\gamma_{\alpha}$ such that $\phi\left(E_{11}-\alpha^{-1} E_{1 j}\right) \equiv E_{11}-\beta_{\alpha}^{-1} E_{1 j}$ and $\phi\left(E_{11}-\alpha^{-1} E_{j 1}\right) \equiv E_{11}-\gamma_{\alpha}^{-1} E_{j 1}$. Define $f_{j}, g_{j}: \mathbb{F} \rightarrow \mathbb{F}$ by $f_{j}(0)=g_{j}(0)=0, f_{j}(\alpha)=\beta_{\alpha}$ and $g_{j}(\alpha)=\gamma_{\alpha}$ for all nonzero $\alpha$.

Let $\alpha \neq 0$. Then $\left(E_{11}+\alpha E_{1 j}\right)\left(E_{11}-\alpha^{-1} E_{j 1}\right)=0$. Hence

$$
\begin{aligned}
\left(1+f_{j}\left(-\alpha^{-1}\right)^{-1} g_{j}(\alpha)^{-1}\right) E_{11} & =\left(E_{11}-f_{j}\left(-\alpha^{-1}\right)^{-1} E_{1 j}\right)\left(E_{11}-g_{j}(\alpha)^{-1} E_{j 1}\right) \\
& =\phi\left(E_{11}+\alpha E_{1 j}\right) \phi\left(E_{11}-\alpha^{-1} E_{j 1}\right)=0 .
\end{aligned}
$$

It follows that $g_{j}(\alpha)=-f_{j}\left(-\alpha^{-1}\right)^{-1}$. 
Next we show that $f_{j}$ is injective. Suppose $f_{j}(\alpha)=f_{j}(\beta)$ for some nonzero $\alpha \neq \beta$. Then

$$
\begin{aligned}
\phi\left(E_{11}-\beta^{-1} E_{1 j}\right) \phi\left(E_{11}+\alpha E_{j 1}\right) & =\left(E_{11}-f_{j}(\beta)^{-1} E_{1 j}\right)\left(E_{11}-g_{j}\left(-\alpha^{-1}\right)^{-1} E_{j 1}\right) \\
& =\left(E_{11}-f_{j}(\alpha)^{-1} E_{1 j}\right)\left(E_{11}+f_{j}(\alpha) E_{j 1}\right)=0 .
\end{aligned}
$$

This is impossible as $\left(E_{11}-\beta^{-1} E_{1 j}\right)\left(E_{11}+\alpha E_{j 1}\right)=\left(1-\alpha \beta^{-1}\right) E_{11} \neq 0$. Thus $f_{j}$ is injective.

The mapping $g_{j}$ is also injective, as $g_{j}(\alpha)=-f_{j}\left(-\alpha^{-1}\right)^{-1}$ for all nonzero $\alpha$.

Now let $A=\left(\begin{array}{lllll}1 & x_{2} & \cdots & x_{n}\end{array}\right)^{t}\left(\begin{array}{llll}1 & y_{2} & \cdots & y_{n}\end{array}\right)$. Then $\phi(A)$ is rank one with nonzero $(1,1)$-th entry so that

$$
\phi(A) \equiv W=\left(\begin{array}{llllll}
1 & u_{2} & \cdots & u_{n}
\end{array}\right)^{t}\left(\begin{array}{llll}
1 & v_{2} & \cdots & v_{n}
\end{array}\right)
$$

for some $u_{2}, \ldots, u_{n}, v_{2}, \ldots, v_{n}$ in $\mathbb{F}$. By Lemma 5.1, $u_{i}=0$ if and only if $x_{i}=0$, and $v_{i}=0$ if and only if $y_{i}=0$. Now if $x_{j} \neq 0$, then $\left(E_{11}-x_{j}^{-1} E_{1 j}\right) A=0$. We have

$$
\left(E_{11}-f_{j}\left(x_{j}\right)^{-1} E_{1 j}\right) W \equiv\left(E_{11}-f_{j}\left(x_{j}\right)^{-1} E_{1 j}\right) \phi(A)=0,
$$

and hence $1-f_{j}\left(x_{j}\right)^{-1} u_{j}=0$ or $u_{j}=f_{j}\left(x_{j}\right)$. Similarly, we get that $v_{j}=g_{j}\left(y_{j}\right)$.

Finally, since $\phi$ satisfies (5.2),

$$
\left(1 f_{2}(1) \cdots f_{n}(1)\right)^{t}\left(1 g_{2}(1) \cdots g_{n}(1)\right) \equiv \phi\left(e e^{t}\right)=(1 \cdots 1)^{t}\left(y_{1} \cdots y_{n}\right)
$$

for some nonzero $y_{1}, \ldots, y_{n} \in \mathbb{F}$. It follows that $1=f_{2}(1)=\cdots=f_{n}(1)$.

ASSERTION 2. For any distinct $i, j>1$, and any $x_{i}, x_{j}, y_{i}, y_{j} \in \mathbb{F}$,

(5.4) $1+x_{i} y_{i}+x_{j} y_{j}=0$ if and only if $1+f_{i}\left(x_{i}\right) g_{i}\left(y_{i}\right)+f_{j}\left(x_{j}\right) g_{j}\left(y_{j}\right)=0$.

Proof. Take $A=E_{11}+x_{i} E_{1 i}+x_{j} E_{1 j}$ and $B=E_{11}+y_{i} E_{i 1}+y_{j} E_{j 1}$. The conclusion follows from (2.2).

ASSERTION 3. For each $i, f_{i}=g_{i}$, and they are multiplicative.

Proof. Take $\left(x_{i}, x_{j}\right)=(\alpha, 1)$ and $\left(y_{i}, y_{j}\right)=(\beta,-\alpha \beta-1)$. By (5.4), we have $1+f_{i}(\alpha) g_{i}(\beta)+g_{j}(-\alpha \beta-1)=0$. Now take $\left(x_{i}, x_{j}\right)=\left(\alpha^{\prime}, 1\right)$ and $\left(y_{i}, y_{j}\right)=$ $\left(\beta^{\prime},-\alpha^{\prime} \beta^{\prime}-1\right)$ with $\alpha^{\prime} \beta^{\prime}=\alpha \beta$. Then $1+f_{i}\left(\alpha^{\prime}\right) g_{i}\left(\beta^{\prime}\right)+g_{j}\left(-\alpha^{\prime} \beta^{\prime}-1\right)=0$. It follows that

$$
1+f_{i}(\alpha) g_{i}(\beta)+g_{j}(-\alpha \beta-1)=0=1+f_{i}\left(\alpha^{\prime}\right) g_{i}\left(\beta^{\prime}\right)+g_{j}\left(-\alpha^{\prime} \beta^{\prime}-1\right),
$$

that is, $f_{i}(\alpha) g_{i}(\beta)=f_{i}\left(\alpha^{\prime}\right) g_{i}\left(\beta^{\prime}\right)$ for any $\alpha \beta=\alpha^{\prime} \beta^{\prime}$. Then for any $\alpha, \beta$,

$$
f_{i}(\alpha) f_{i}(\beta) g_{i}(1)=f_{i}(\alpha) f_{i}(1) g_{i}(\beta)=f_{i}(\alpha) g_{i}(\beta)=f_{i}(\alpha \beta) g_{i}(1) .
$$


Since $g_{i}(1) \neq 0, f_{i}$ is multiplicative.

Since $f_{i}$ is injective and multiplicative, $f_{i}(-1)=-1$, and so

$$
g_{i}(1)=-f_{i}\left(-1^{-1}\right)^{-1}=1 .
$$

Thus, $g_{i}(\alpha)=g_{i}(\alpha) f_{i}(1)=g_{i}(1) f_{i}(\alpha)=f_{i}(\alpha)$ for all $\alpha \in \mathbb{F}$.

ASSERTION 4. $f_{2}=\cdots=f_{n}=g_{2}=\cdots=g_{n}$.

PROOF. It suffices to prove that $f_{2}=f_{j}$ for any $j \geq 2$. Take $\left(x_{2}, x_{j}\right)=(-\alpha-1, \alpha)$ and $\left(y_{2}, y_{j}\right)=(1,1)$ in (5.4). Then we have

$$
1+f_{2}(-\alpha-1)+f_{j}(\alpha)=1+f_{2}(-\alpha-1) g_{2}(1)+f_{j}(\alpha) g_{j}(1)=0 .
$$

Hence $f_{j}(\alpha)=-f_{2}(-\alpha-1)-1=f_{2}(\alpha+1)-1$. In particular, $1=f_{j}(1)=f_{2}(2)-1$, that is, $f_{2}(2)=2$. Interchanging the roles of $x_{2}$ and $x_{j}$, we get $f_{2}(\alpha)=f_{j}(\alpha+1)-1$ and $f_{j}(2)=2$. Then

$$
\begin{aligned}
f_{j}(\alpha)-1 & =f_{2}(\alpha-1)=f_{2}(2) f_{2}((\alpha-1) / 2)=2\left[f_{j}((\alpha-1) / 2+1)-1\right] \\
& =f_{j}(2) f_{j}((\alpha-1) / 2+1)-2=f_{j}(\alpha+1)-2,
\end{aligned}
$$

that is, $f_{j}(\alpha+1)=f_{j}(\alpha)+1$. Thus, $f_{2}(\alpha)=f_{j}(\alpha+1)-1=f_{j}(\alpha)$.

ASSERTION 5. The mappings $f_{2}=\cdots=f_{n}=g_{2}=\cdots=g_{n}$ are additive.

PROOF. For any nonzero $\alpha, \beta \in \mathbb{F}$ such that $\alpha+\beta \neq 0$, let

$$
\left(x_{2}, x_{3}\right)=(-\alpha /(\alpha+\beta),-\beta /(\alpha+\beta)) \text {. }
$$

Then with $\left(y_{2}, y_{3}\right)=(1,1)$, we have

$$
\begin{aligned}
& 1-f_{2}(\alpha+\beta)^{-1} f_{2}(\alpha)-f_{2}(\alpha+\beta)^{-1} f_{2}(\beta) \\
& \quad=1+f_{2}(-\alpha /(\alpha+\beta))+f_{3}(-\beta /(\alpha+\beta))=0 .
\end{aligned}
$$

Thus, $f_{2}(\alpha+\beta)=f_{2}(\alpha)+f_{2}(\beta)$, that is, $f_{2}$ is additive.

ASSERTION 6. There exists a $\sigma_{1}$ such that (5.3) holds.

PROOF. Let $\sigma_{1}=f_{2}=\cdots=f_{n}=g_{2}=\cdots=g_{n}$. Then by the above assertions, $\sigma_{1}$ is a field monomorphism on $\mathbb{F}$ and $\phi(A)=\left(\sigma\left(a_{i j}\right)\right)$ for all $A=\left(a_{i j}\right) \in \mathcal{M}_{n}^{1}$ with $a_{11}=1$. Now for any $B=\left(b_{i j}\right) \in \mathcal{M}_{n}^{1}$ with $b_{11} \neq 0$, we have that $B \equiv\left(1 / b_{11}\right) B$. By Lemma 2.3,

$$
\phi(B) \equiv \phi\left(\left(1 / b_{11}\right) B\right) \equiv\left(\sigma\left(b_{i j} / b_{11}\right)\right) \equiv \sigma\left(b_{11}^{-1}\right)\left(\sigma\left(b_{i j}\right)\right) \equiv\left(\sigma\left(b_{i j}\right)\right) .
$$

The assertion follows. 
Suppose $n \geq 3$. By Lemma 5.3, there exist an invertible $S \in \mathcal{M}_{n}$, and a field monomorphism $\sigma$ of $\mathbb{F}$ such that

$$
\phi(A) \equiv S A_{\sigma} S^{-1}
$$

for all rank one matrix $A$ in $\mathcal{S}$ with nonzero trace. Now, suppose $B=x y^{t} \in \mathcal{S} \backslash\{0\}$ has zero trace. Let $u, v \in \mathbb{F}^{n}$ be such that $u^{t} x=y^{t} v=1$. Then (5.5) holds for $A=x u^{r}$ and $A=v y^{t}$. By Lemma 2.3, $R(\phi(B))=R\left(\phi\left(x u^{t}\right)\right)$ and $N(\phi(B))=N\left(\phi\left(v y^{t}\right)\right)$. Hence, $\phi(B) \equiv S B_{\sigma} S^{-1}$. Combining the above arguments, we see that for each rank one $A$, we have $\phi(A)=\lambda_{A} S A_{\sigma} S^{-1}$ for some nonzero $\lambda_{A}$. Define $\mu: \mathcal{S} \cap \mathcal{M}_{n}^{1} \rightarrow \mathbb{F}^{*}$ by $\mu(A)=\lambda_{A}$. The desired conclusion follows.

\section{Acknowledgements}

We would like to thank Dr. Wai-Shun Cheung, Professor Jianlian Cui, Professor Jinchuan Hou, and Professor Yiu-Tong Poon for inspiring discussions. Professors Cui and Hou have extended some of our results to standard operator algebras with the surjective assumption on $\phi$. We thank them for sending us their preprint. Furthermore, we thank Professor Peter Šemrl for many helpful comments on an earlier draft of the paper.

\section{References}

[1] B. Alpers and E. M. Schröder, 'On mappings preserving orthogonality of nonsingular vectors', J. Geom. 41 (1991), 3-15.

[2] R. Bhatia, P. Šemrl and A. Sourour, 'Maps on matrices that preserve the spectral radius distance', Studia Math. 134 (1999), 99-1 10.

[3] M. A. Chebotar, W.-F. Ke, P.-H. Lee and N.-C. Wong, 'Mappings preserving zero products', Studia Math. 155 (2003), 77-94.

[4] J. Cui and J. Hou, 'Non-linear numerical radius isometries on atomic nest algebras and diagonal algebras', J. Funct. Anal. 206 (2004), 414-448.

[5] G. Dolinar and P. Šemrl, 'Determinant preserving maps on matrix algebras', Linear Algebra Appl. 348 (2002), 189-192.

[6] J. Hou, 'Maps preserving numerical range of operator products and Jordan triple products', presentation at the mini-workshop on preserver problems, University of Hong Kong, July, 2004.

[7] J. Hou and $X$. Zhang, 'Ring isomorphisms and linear or additive maps preserving zero products on nest algebras', Linear Algebra Appl. 387 (2004), 343-360.

[8] L.-K. Hua, 'A theorem on matrices over a sfield and its applications', J. Chinese Math. Soc. (N.S.) 1 (1951), 109-163.

[9] H. Kestelman, 'Automorphisms of the field of complex numbers', Proc. London Math. Soc. (2) 53 (1951), 1-12.

[10] C.-K. Li, 'C-numerical ranges and $C$-numerical radii', Linear and Multilinear Algebra 37 (1994), $51-82$. 
[11] C.-K. Li and S. Pierce, 'Linear preserver problems', Amer. Math. Monthly 108 (2001), 591-605.

[12] L. Molnár, 'Orthogonality preserving transformations on indefinite inner product spaces: generalization of Uhlhorn's version of Wigner's theorem', J. Funct. Anal. 194 (2002), 248-262.

[13] - 'Some characterizations of the automorphisms of $B(H)$ and $C(X)$ ', Proc. Amer. Math. Soc. 130 (2002), 111-120.

[14] _ - 'Local automorphisms of operator algebras on Banach spaces', Proc. Amer. Math. Soc. 131 (2003), 1867-1874.

[15] S. Pierce, editor of the special issue on 'A survey of linear preserver problems', Linear and Multilinear Algebra 33 (1992).

[16] V. Tan and F. Wang, 'On determinant preserver problems', Linear Algebra Appl. 369 (2003), 311-317.

[17] U. Uhlhorn, 'Representation of symmetry transformations in quantum mechanics', Ark. Fys. 23 (1963), 307-340.

[18] P. Šemrl, 'Linear mappings preserving square-zero matrices', Bull. Austral. Math. Soc. 48 (1993), 365-370.

[19] — 'Applying projective geometry to transformations on rank-one idempotents', $J$. Funct. Anal. 210 (2004), 248-257.

[20] _ _ 'Maps on matrix spaces', Taussky-Todd Lecture, International Linear Algebra Society Conference, Coimbra, Portugal, 2004.

[21] — 'Maps on idempotents', preprint.

[22] P. B. Yale, 'Automorphisms of the complex numbers', Math. Mag. 39 (1966), 135-141.

Department of Mathematics

The University of Hong Kong

Pokfulam Road

Hong Kong

e-mail: jtchan@hku.hk
Department of Mathematics

College of William and Mary

Williamsburg, VA 23187-8795

USA

e-mail: ckli@math.wm.edu

\author{
Department of Mathematics \\ The University of Hong Kong \\ Pokfulam Road \\ Hong Kong \\ and \\ Department of Mathematics \\ University of Connecticut \\ Storrs, CT 06269-3009 \\ USA \\ e-mail: sze@math.uconn.edu
}

